\title{
Review
}

\section{Towards the Understanding of Ice Crystal-Graupel Collision Charging in Thunderstorm Electrification}

\author{
Yuanping $\mathrm{He}^{1,4}$, Boyan $\mathrm{Gu}^{2}$, Daizhou Zhang3, Weizhen Lu' ${ }^{4}$, Chuck Wah $\mathrm{Yu}^{1,5}$, Zhaolin Gu $\mathbf{u}^{1, *}$ \\ 1 Department of Earth and Environmental Sciences, Xi'an Jiaotong University, Xi'an 710049, China \\ 2 Department of Atmospheric Sciences, Texas A\&M University, College Station, TX77840, USA \\ 3 Faculty of Environmental \& Symbiotic Sciences, Prefectural University of Kumamoto, Kumamoto 862- \\ 8502, Japan \\ 4 Department of Architecture and Civil Engineering, City University of Hong Kong, Kowloon Tong, \\ HKSAR, Hong Kong \\ 5 International Society of the Built Environment (ISBE), Milton Keynes MK78HQ, UK \\ * Correspondence: guzhaoln@mail.xjtu.edu.cn; Tel.: +86(029) 83395100
}

\begin{abstract}
Thunderstorm electrification has been studied for hundreds of years. Several mechanisms have been proposed to elucidate the electrification, including convective charging, inductive precipitation charging, and ice crystal-graupel collision charging. Field observations and model studies have demonstrated the vital roles that graupel and ice crystals play in the electrification, but the mechanism of the collision charging is still unclear. The fundamental essence of relative growth rate theory used for explaining the tripole charge structure in thunderclouds also needs a further exploration. We analyze the processes of ice crystal-graupel collision charging from charge migration inside hydrometeors to charge separation between two hydrometeors. The driving effects of temperature gradient and chemical potential gradient in charge migration are clarified, as well as the applicability of the relative growth rate theory, thermoelectric effect and surface tension gradient in different humidities. Based on the understanding from these electrification mechanisms, we propose that the essence of charge separation is driven by non-thermal equilibrium, and future studies on thunderstorm electrification should focus on the dynamical non-thermal equilibrium of cloud particles.
\end{abstract}

Keywords: Thunderstorm electrification; Ice crystal-graupel collision; Relative growth rate theory; Temperature gradient; Non-thermal equilibrium; Tripole charge structure; Thunderclouds hydrometeors

\section{Introduction}

The electrification of thunderclouds usually occur in the zone filled with water droplets, ice crystals and graupel particles in the clouds [1]. These cloud particles (also called hydrometeors) have different weights and structures and can acquire different charges via poly-phase interfacial interactions that lead to charge transfer along with or without mass transfer. Several mechanisms of thunderstorm electrification have been proposed, of which three continue to receive support: convective charging [2] ; inductive precipitation charging []]; and ice crystal-graupel collision charging [4]. The last one is also known as non-inductive precipitation charging. Observations and model studies have supported the hypothesis that graupel and ice crystals play the most vital role in the electrification and lightning of thunderstorms [5-12]. As the majority of hydrometeors in cloud, ice crystals and graupel, which are usually different in weight and temperature, are more likely to collide to result in the transfer of charges between them in convective thunderclouds, i.e., the ice crystal-graupel collision charging. 
The classic tripole charge structure of thunderstorms has been supported by a wide variety of observations [13-17]. Cloud temperature and liquid water content of the environment are the two major parameters in controlling the polarity of charge acquired by large precipitation particles. The crystal-graupel collision charging model proposed by Jayaratne and Saunders is used to explain the tripole charge structure (referring to section 3). Ice crystals are charged positively through collisions with graupel at altitudes above the charge reversal temperature; and charged negatively at altitudes below the reversal temperature [1]. Due to the influence of the gravitation and convection [19], charged particles are redistributed and a tripole charge structure is formed in vertical direction.

The mechanism based on the relative growth rate of cloud particles has been proposed to explain the charge reversal [20], where the vapour-grown crystals grow faster than graupel and positively at colder temperature charge, while graupel grow faster and charge negatively at warmer temperatures. However, how the relative growth rates of these cloud particles vary with humidity and temperature, and how the charge polarity of particles relates to the growth rates are unclear. Although the thermoelectric effect [21] and surface tension gradient [22] have also been used to explain charge transfer in particle collisions, their applicability is limited to lower and higher humidity in atmospheric conditions, respectively.

In this perspective study, we focus on the clarification of the mechanism of charge transfer in cloud particle collisions and the formation of the tripole charge structure in thunderclouds. Section 2 examines the existing models for charge transfer in cloud particles, from charge migration inside hydrometeors to charge separation between two hydrometeors. Section 3 shows further understanding of the crystal-graupel collision charging, resulting in the formation of tripole charge structure. It starts with an investigation of the variation of the growth rates of crystal and graupel in vertical direction with temperature and humidity in thunderclouds, followed by a combination of the thermoelectric effect and the surface tension gradient mechanism in thunderstorms. Other factors, including particle size, particle shape and the relative velocity of colliding particles, that may influence the charge transfer between cloud particles are also considered. This section closes with a discussion of non-thermal equilibrium, suggesting that the non-thermal equilibrium of hydrometeors is very likely the fundamental cause of cloud particle charging. Finally, the conclusions and perspectives from this work are presented in section 4 .

\section{Clarification of charge transfer in cloud particles}

Ice crystals and graupel as the main hydrometeor particles in clouds usually have different weights and temperatures, and frequently collide, especially under the effect of strong convection in thunderstorms. Such hydrometeor particles would be charged after their collision, contact and separation. However, in the following, ice crystals and graupel are regarded as different sized particles, that is small ice particle and large graupel particle; and the temperature difference inside cloud particle is especially concerned, together with temperature differences between particles and their surrounding environment. To demonstrate the electrification mechanisms of particle collision, charge transfer between colliding particles is divided into two steps: charge migration inside particles and charge separation between particles.

\subsection{Charge migration inside hydrometeors}

The electrification of thunderclouds mainly occurs in the zone where all kinds of hydrometeors coexist, primarily related to the separation of $\mathrm{OH}^{-}$ions from $\mathrm{H}^{+}$ions. Water is the most common and most abundant condensable substance in the earth system, and hence the electrification of $\mathrm{H}_{2} \mathrm{O}$ clouds is considered to be the main process of charge generation. Ion migration inside hydrometeors, along with charge migration between hydrometeors, is driven by the temperature gradient and chemical potential gradient [23] . 


\subsubsection{Temperature gradients inside hydrometeors}

Temperature gradient drives charge migration due to the thermoelectric effects on ions mobility. It is assumed that a spherical water droplet in a non-thermal equilibrium state would generate a concentration gradient of ions inside the particle. The flux of an ion, $N_{i}$, goes from a region of high concentration to a region of low concentration inside this particle, as defined by Eq. (1) [24]:

$$
N_{i}=-D_{i} \frac{\partial c_{i}}{\partial R} \propto-D_{i} \frac{\partial T}{\partial R}
$$

where $R$ is the particle radius and $D$ is the diffusion coefficient $\left(\mathrm{m}^{2} \mathrm{~s}^{-1}\right) . D_{i}$ is proportional to the squared velocity of the diffusing ions, and depends on the temperature $T$, viscosity of the fluid $\mu$, and size of the ion $r_{i}$. It is defined by Eq. (2):

$$
D=\frac{\kappa T}{6 \pi \mu r_{i}}
$$

where $c_{i}$ denotes the concentration of the ion $(\mathrm{mol} \mathrm{m}-3), \kappa$ is the Boltzmann constant, $1.38 \times 10^{-23} \mathrm{~J} \mathrm{~K}^{-1}$, and $i$ denotes $\mathrm{H}^{+}$or $\mathrm{OH}^{-}$.

Since the ionic radius of $\mathrm{H}^{+}$is less than that of $\mathrm{OH}^{-}$, the migration flux of $\mathrm{H}^{+}$is higher than that of $\mathrm{OH}^{-}$under the same temperature gradient. In addition, the degree of water dissociation increases with the average temperature of particle and the temperature gradient inside particle. As a result, more $\mathrm{H}^{+}$ions would accumulate in regions of lower temperature in the particle. Especially in ice particles, the diffusion coefficient $D_{i}=u \kappa T / e$, or the mobility $u$, of the $\mathrm{H}^{+}$ion is at least ten times greater than that of the $\mathrm{OH}^{-}$ion [25]. If an ice particle moves to an environment with supersaturated water vapour, the particle would grow and its ice-vapour interface region would acquire an excess of negative charge due to the release of latent heat. In contrast, if an ice particle moves to a drier environment, the sublimation happens on the surface of particle and then cools the particle surface, and thus the polarity of surface charge would be reversed (Fig. 1).

(a) Condensation

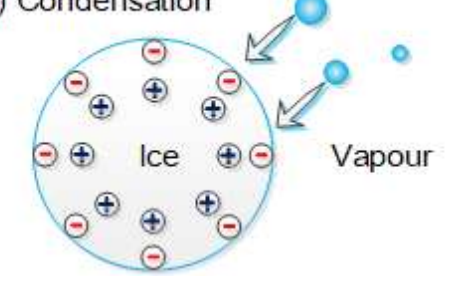

(b) Sublimation

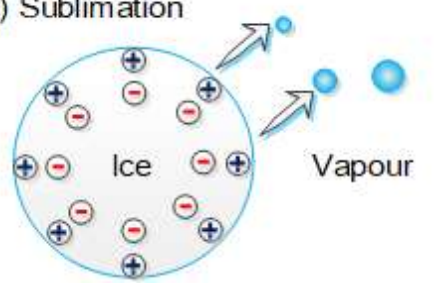

Figure 1. Charge migration inside an ice particle driven by a different temperature gradient. (a) When an ice particle is growing by vapour diffusion, the latent heat of condensation would heat the particle surface to a higher temperature than the temperature inside the particle, and the particle surface would accumulate a net negative charge; (b) When the ice particle is introduced into a drier environment, the temperature of the particle surface would be lower than the temperature inside the particle because of sublimation, and the particle surface would accumulate a net positive charge.

According to calculations and experimental tests from Latham and Mason [25, 26] $]$, a potential difference of magnitude $V_{T}=2 \Delta \mathrm{T} \mathrm{mV}$ would be developed when a steady temperature difference $\Delta T$ is maintained between a particle surface and its inside. Assume that an ice particle is in the mature phase of a thundercloud with a strong updraft of $10 \mathrm{~m} \mathrm{~s}^{-1}$ [27]. For an ice particle with radius of $1.0 \times 10^{-}$ ${ }^{4} \mathrm{~m}$, it would be quickly lifted by the updraft speed and the temperature on its surface declines linearly with elevation through the troposphere at a rate of approximately $0.6^{\circ} \mathrm{C}$ for $100 \mathrm{~m} \mathrm{[28}$ ]. Therefore, during $\Delta t=0.1 \mathrm{~s}$, their rising distances are $\Delta h=1 \mathrm{~m}$, and their temperature variations are $\Delta T=0.006^{\circ} \mathrm{C}$. Thus, there is a potential differential between the particle surface and its inside, $V_{T}=$ $1.2 \times 10^{-5} \mathrm{~V}$. 


\subsubsection{Chemical potential gradient}

Charge migration driven by a chemical potential gradient is based on the criterion of spontaneity, in which an ion migrates spontaneously when its Born energy is smaller than zero. For an ice particle in the air, the density of water molecules within the interfacial region is less than that inside the ice.

The condition for chemical equilibrium is as given by Eq. (3):

$$
\mu_{\mathrm{H}_{2} \mathrm{O}}=\mu_{\mathrm{H}^{+}}+\mu_{\mathrm{OH}^{-}}
$$

where the chemical potentials can be represented by Eq. (4), as

$$
\mu_{\mathrm{H}^{+}}=\mu_{\mathrm{H}^{+}}^{0}+\kappa T \ln C_{\mathrm{H}^{+}}
$$

and Eq. (5):

$$
\mu_{\mathrm{OH}^{-}}=\mu_{\mathrm{OH}^{-}}^{0}+\kappa T \ln C_{\mathrm{OH}^{-}}
$$

It is assumed that the bulk ice phase is at equilibrium with the interfacial region. Due to a reduction in the ice density, the dielectric constant of the interfacial region will correspondingly reduce. The chemical potential of an ion in this region will, therefore, increase by an amount known as the Born energy. High chemical potential at an interfacial region (denoted by region 1) can cause ions to transfer from the region 1 with a dielectric constant $\varepsilon_{1}$ to a region with a larger dielectric $\varepsilon_{2}$ (denoted by region 2), and the expression is given by Eq. (6), [29]]:

$$
\Delta \mu_{i}(\text { Born })=-\frac{28 z^{2}}{r_{i}}\left[\frac{1}{\varepsilon_{1}}-\frac{1}{\varepsilon_{2}}\right] \kappa T \quad(T<300 \mathrm{~K})
$$

where $\mu_{i}$ is the chemical potential $\left(\mathrm{J} \mathrm{mol}^{-1}\right) ; z$ is the valence number of ion; $r_{i}$ is the ionic radii $(\mathrm{nm}) ; \kappa$ is the Boltzmann constant, $1.38 \times 10^{-23} \mathrm{~J} \mathrm{~K}^{-1} ; T$ is the thermodynamic temperature $(\mathrm{K})$. Since the Born energy is inversely proportional to the ionic radii, and the ionic radius of $\mathrm{H}^{+}$ions is less than that of $\mathrm{OH}^{-}$ions, the following inequality applies whenever $\varepsilon_{1}<\varepsilon 2: \Delta \mu_{\mathrm{H}^{+}}<\Delta \mu_{\mathrm{OH}^{-}}<0$. Due to the difference in the chemical potential gradient between $\mathrm{H}^{+}$and $\mathrm{OH}^{-}$, more $\mathrm{H}^{+}$ions would move from the particle surface to inner part of the particle, and an excess of negative charges would accumulate within the interfacial region, as shown in Fig. 2.

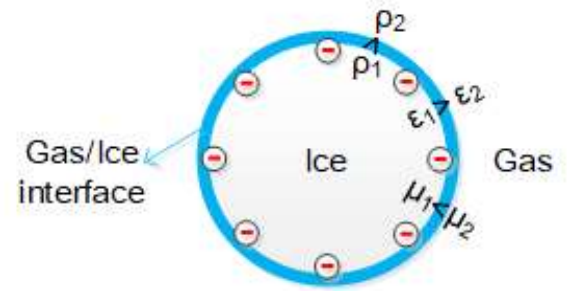

Figure 2. Charge migration inside an ice particle driven by a potential gradient. The particle surface accumulates a net negative charge.

The electrical potential, $V_{c}$, caused by radial chemical potential gradients of hydrogen and hydroxyl ions inside an ice particle, can be approximated by Eq. (7), as [299]:

$$
V_{C}=\frac{1}{Q}\left[\Delta \mu_{\mathrm{OH}^{-}}(\text {Born })-\Delta \mu_{\mathrm{H}^{+}}(\text {Born })\right]
$$

The ionic radii of $\mathrm{H}^{+}$ion and $\mathrm{OH}$-ion are $1.2 \times 10^{-12} \mathrm{~m}$ and $1.76 \times 10^{-10} \mathrm{~m}$, respectively [29]. The value of vacuum permittivity $\varepsilon_{0}$ is $8.85 \times 10^{-12} \mathrm{C}^{2} \mathrm{~J}^{-1} \mathrm{~m}^{-1}$. The permittivity constants for air and ice are 1 and 4 , respectively. Here, the permittivity constant of ice/air interface is assumed as 2 . The surface charge density could be estimated by $\sim 4 \times 10^{-5} \mathrm{C} \mathrm{m}^{-2}$ [22] . For an ice particle with a radius of $1.0 \times 10^{-4} \mathrm{~m}$, the 
charge on particle surface is $3.2 \times 10^{-10} \mathrm{C}$. From Eq. (6) and Eq. (7), the electrical potential $V_{C}$ is obtained, with value of $0.98 \times 10^{-12} \mathrm{~V}$.

\subsubsection{Comparison of charge migration mechanisms}

Except for the temperature gradient and chemical potential gradient, the electrical double layer and dislocations in ice structure were explained by electrical mechanisms due to the presence of the net negative/ positive charges on the particle surface base on the following observations [27]: (i) An electrical double layer is defined as a dipolar layer just inside an interface between water and air, ice and air, or water and ice. It was thermodynamically advantageous for molecules at the surface of pure water in air to be oriented with their negative vertices outward [30]. Theoretical and experimental studies have shown that ice in equilibrium with the vapour has a disordered quasiliquid layer at the ice-vapour interface and there is net negative charge on the surface [31]. The thickness of this layer increases with temperature and also with growth rate from the vapour. In fact, the presence of a net negative charge in the quasi-liquid layer on ice is due to temperature and chemical factors. (ii) A dislocation is defined as a line defect in a crystal which disrupts the otherwise ideal arrangement of atoms or molecules [32]. Contrary to liquid-like layer, the negative charge at water-vapour interface due to electrical double layer, the dislocations would carry positive charge. The density of dislocations increase monotonically with the growth rate of the ice crystal [33]. The dislocation concentration in rim ice increased as deposit temperature is decreased. Thus, the dislocation density is a function of the rime temperature and the droplet freezing time [27]. Therefore, the dislocation theory of charge transfer implies that the dislocation depends on the temperature and vapour pressure on particle surfaces. The charge migration inside particles is mainly attributed to the nonhomogeneous distribution of temperature and chemical potential.

Furthermore, the electrical potential caused by temperature gradient is several orders higher than that caused by physical potential gradient. For a hydrometeor in thermal equilibrium, the accumulation of net negative charges on the particle surface is small if the accumulation is driven by the chemical potential gradient only. The typical argument is numerous rain events, where chemical effects always exist, and these are generally accompanied no thunder and lighting [34]. For a hydrometeor in non-thermal equilibrium state, the impact of the chemical potential gradient on charge migration would be enhanced in that the chemical potential is proportional to temperature. Therefore, the temperature gradient itself plays a vital role in charge migration of cloud particles in thunderclouds.

\subsection{Charge separation between hydrometeors}

\subsubsection{Thermoelectric effect}

The thermoelectric effect is based on a temperature gradient, as discussed above. For two hydrometeors whose surfaces have accumulated $\mathrm{H}^{+}$and $\mathrm{OH}^{-}$, their internal energy and temperature will vary during collision, generating frictional heat. Different temperature increments will result in different water ionizations and thus different $\mathrm{H}^{+} / \mathrm{OH}^{-}$ion concentrations on the surfaces of hydrometeors. A temperature increase of a hydrometeor will increase $\mathrm{H}^{+}$and $\mathrm{OH}^{-}$concentrations on its surface. Although the migrations of $\mathrm{H}^{+} / \mathrm{OH}^{-}$from the high temperature hydrometeor to the low temperature hydrometeor can occur simultaneously, the higher mobility of $\mathrm{H}^{+}$than that of $\mathrm{OH}^{-}$will make the entire high-temperature hydrometeor negatively charged and low-temperature hydrometeors positively charged.

It is generally assumed that the internal energy conversion is equally partitioned between two colliding ice particles, so for two spherical particles with different sizes, the temperature increment of the small particle is higher than that of the larger one after their collision, according to Eq. (10) of the temperature increment [35]: 


$$
\Delta T_{a}=\frac{\frac{1}{2} W}{\frac{4}{3} \pi R^{3} \rho_{p} c_{p}}
$$

where $W$ is the internal energy released in the collision; $\rho_{p}$ is the particle density; and $c_{p}$ is the specific heat capacity. $\Delta T_{a}$ is inversely proportional to the third power of the particle radius. Therefore, for two different sized ice particles with the same initial temperature, the small particle with higher temperature acquires negative charge and the large particle with lower temperature acquires positive charge after collision and separation [36], as shown in Fig. 3.

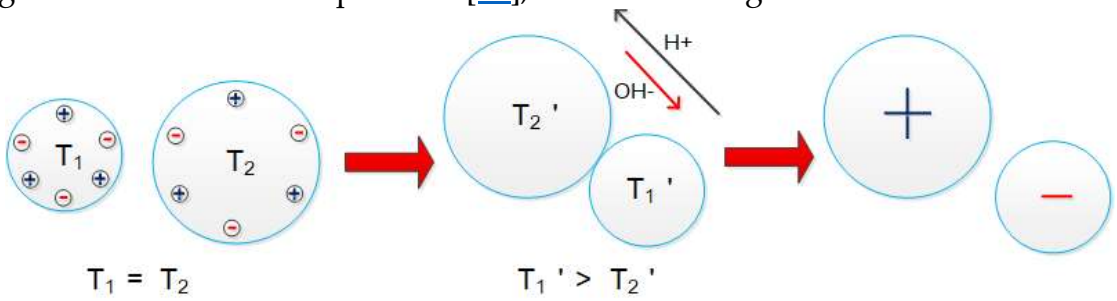

Figure 3. $\mathrm{H}+/ \mathrm{OH}-$ dynamic transfer between two different sized ice particles.

In addition, assume two ice particles are completely covered with water films formed from vapour condensation and the thicknesses of the films are the same. This is common for two ice particles at the same altitude, where the mass increment of water film due to the flux of water vapour by molecular diffusion is proportional to the particle surface area [37]. The temperature increment of particle due to the release of the latent heat can be defined by Eq. (11), as:

$$
\Delta T_{\mathrm{b}}=\frac{Q_{w} \rho_{w} \cdot 4 \pi R^{2} l}{c_{p} \rho_{p} \cdot \frac{4}{3} \pi R^{3}}=\frac{3 Q_{w} l}{c_{p} R} \cdot \frac{\rho_{w}}{\rho_{p}},
$$

where $Q_{w}$ is the condensation heat of water, and $l$ is the thickness of the water film. In this situation, $\Delta T_{b}$ is inversely proportional to the particle radius. Therefore, for two different sized ice particles with the same initial temperature $T_{1}=T_{2}$, the small particle attains a higher temperature after vapour condensation to the same film thickness, that is, $T_{1}^{\prime}>T_{2}^{\prime}$. According to the thermoelectric effect, the small particle acquires negative charge and the large particle acquires positive charge after collision and separation, as shown in Fig. 4.

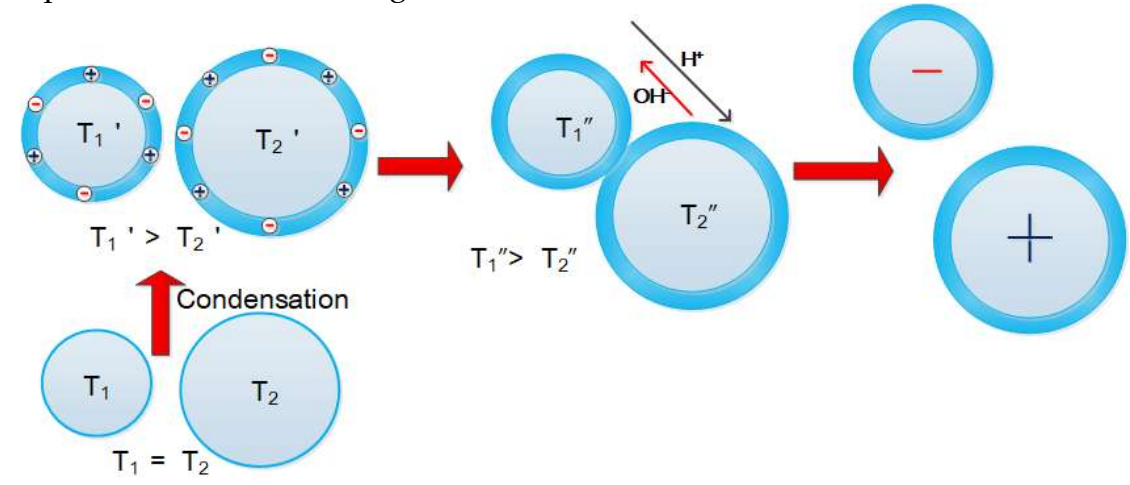

Figure 4. $\mathrm{H}^{+} / \mathrm{OH}^{-}$dynamic transfer between two different sized ice particles with an equally thick film formed from vapour condensation.

Furthermore, ice particles carrying $\mathrm{H}^{+} / \mathrm{OH}^{-}$ions in different concentrations because of different initial temperatures, are common in clouds and related to their growth histories. The ion concentration differential (or say, surface potential differential [38] would promote charge transfer 
between two particles once they collide [39]. For ice crystal-graupel collision, the riming graupel, usually with a higher temperature, would acquire a net negative charge after collision according to the thermoelectric effect. However, if one high temperature particle, which has accumulated large amounts of net negative charge on its surface, contacts and collision with another particle, more $\mathrm{OH}^{-}$ would be transferred from the high temperature particle to low temperature particle due to ion diffusion, resulting in the high temperature particle becoming more positively charged.

\subsubsection{Surface tension gradient}

Graciaa et al. [22] introduced the idea that the surface tension gradient, due to temperature differential, is a driving force behind the transport of excess negative charge from the warmer hydrometeor to the colder body, yielding a separation of charge. As shown in Fig. 5, an ice crystal strikes a water-covered target surface, creating a surface tension gradient that generates a flow directly from the water-covered target surface to the cold crystal, leaving the target with a net positive charge.

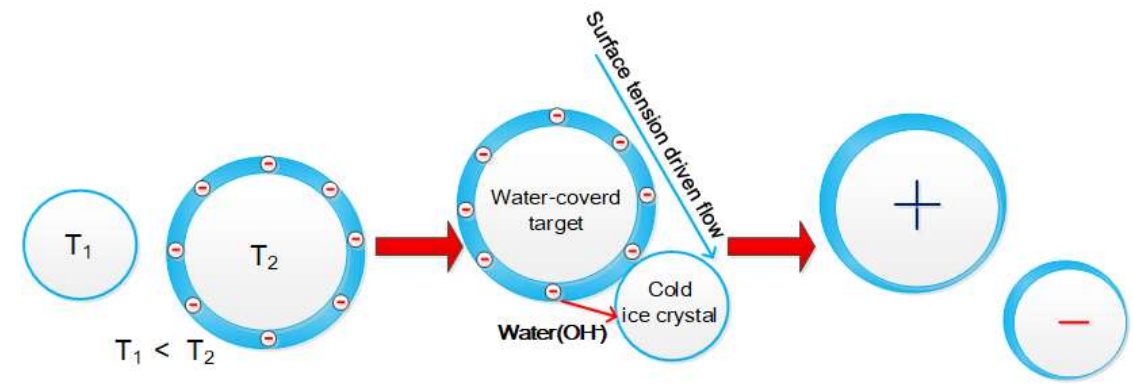

Figure 5. The surface layer of a thick water film is drawn towards a cold rebounding ice crystal. Negative charge is, therefore transferred along with the water film.

The surface tension gradient, acting along an air-water interface, creates an interfacial flow directly from the region of lower interfacial tension (higher temperature) to the region of higher interfacial tension (lower temperature). As a result, the colder hydrometeor would carry a negative charge and the warmer a positive charge. This appears to contradict with the thermoelectric effect, where colder hydrometeors generally acquire a positive charge. In fact, the results proposed by Gracia [22] are all premised on the outer layer of a water droplet being negatively charged, which means that the surface tension gradient mechanism is more applicable in wet growth regions with higher cloud water content. Under such a region, at least one of the surfaces of the colliding hydrometeors contains a thick water film, but the thermoelectric mechanism is dominant in drier regions having less liquid water, or when two hydrometeors have the same growth rates.

\subsection{Non-thermal equilibrium of hydrometeors}

Cloud particles differ remarkably in size in space and time, and are frequently present in a wide size range of $1-1000 \mu \mathrm{m}$ in the mixed-phase region of clouds [40]. Cloud particles in thunderstorms will shuttle between different environments under strong convection. To illustrate the importance of non-thermal equilibrium ionization of cloud particles in thunderstorm electrification, we compared the response time and thermal relaxation time of an ice particle which comes into a new environment.

It is assumed that, if an ice particle, initially growing under the conditions near ice saturation and having an equilibrium temperature below the temperature of particles growing at water saturation, gets into a new environment that is at or above water saturation, the thermal equilibrium of the particle will be broken. To get to a new thermal equilibrium, the particle surface experiences an enhanced growth rate because the lower surface temperature reduces the equilibrium surface vapour pressure over the ice surface. This rapid growth increases the surface temperature of the particle due to the release of latent heat, ultimately increasing the surface vapour pressure [9].

The characteristic time for vapour diffusion on an ice particle surface to respond to a new local environment can be determined by Eq. (12) as $[\underline{41}, \underline{42}]$ 


$$
t_{r}=\frac{R^{2}}{\pi D_{V}}
$$

where $R$ is the particle radius and $D_{V}$ is the diffusion coefficient for water vapour. For small ice particle, the respond time is only in the order of few microseconds, but it takes tens of milliseconds to reach thermal equilibrium in the new environment $[\underline{9}, \underline{43}]$. Thus, the ice particle, maintaining a high growth rate, will accumulate more negative charge on its surface under the effect of a temperature gradient and will acquire a positive charge after colliding with another target particle.

The droplets remain in a supercooled liquid state until they contact an ice surface, whereupon they freeze and stick to the surface on a process called riming. Alternatively, the supercooled droplets freeze spontaneously at sufficiently low temperature. The rate of freezing depends on the heat transfer to the environment, and the thermal relaxation time for this kind of cloud particles is up to several seconds [44]. Since the direction of temperature gradient inside the droplet is the reverse of that of the foregoing crystal, the charges will be opposite each other after collision and separation.

Almost all particles at the updraft-downdraft transition zone of thunderstorms are in nonthermal equilibrium states. Non-thermal equilibrium inside particles has been commonly ignored in studies of precipitation and thunderstorms, and the non-thermal equilibrium ionizations inside particles provides a new viewpoint for the elucidation of the complexity of thunderstorm electrification.

\section{Further understanding of thunderstorm electrification}

The crystal-graupel collision charging model proposed by Jayaratne and Saunders to explain the tripole charge structure is shown in Fig. 6. Ice crystals charge positively through collisions with graupel at altitudes above the charge reversal temperature; and charge negatively at altitudes below the reversal temperature.

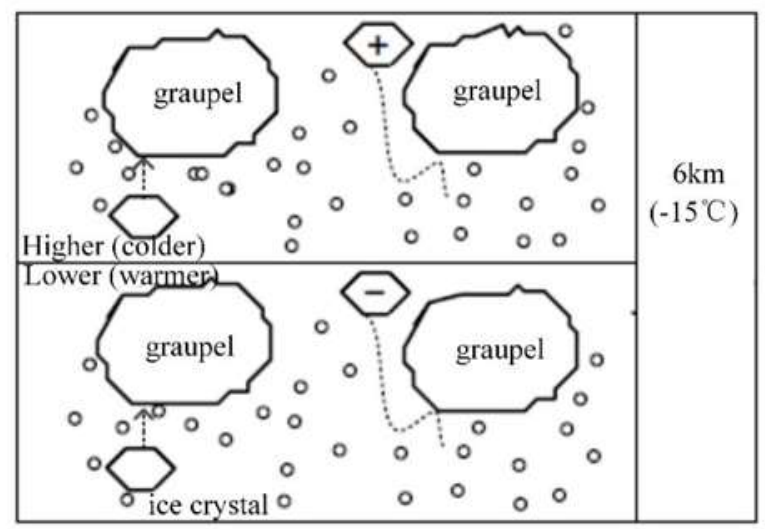

Figure 6. Charge transfer by collisions in the ice crystal-graupel mechanism of cloud electrification, presumably at $6 \mathrm{~km}$ with the reversal temperature $-15^{\circ} \mathrm{C}[\underline{18}]$.

\subsection{Relative growth rate theory}

The relative growth rate theory holds that during ice crystal-graupel collision, the particle that grows faster would be charged positively [ㄴ5]. It has been supposed that ice crystals usually grow faster than graupel particles above the altitude of the reversal temperature and grow slower below the altitude, creating the so-called tripole charge structure of the clouds (Fig. 6).

In fact, in the lower (warmer) region of thunderclouds, the surface of a riming graupel grows directly from the vapour in the cloud, as well as from the vapour supplied by droplets freezing upon the surface. The time required for freezing is longer at warm temperatures. During this time, the 
surrounding ice surface would grow rapidly from the vapour supplied by the freezing water. Hence, the surface of a riming graupel would grow faster because the vapour is warmer and has a higher liquid water content, causing it to become positively charged according to the relative growth rate theory.

In the upper (colder) region, ice crystals may grow faster on average and charge positively, while graupel would charge negatively at colder temperatures. In the cloud, graupel pellets will be warmer than ice crystals due to the release of latent heat from accreted droplets. Given a high enough accretion rate, the rimer surface temperature approaches $0{ }^{\circ} \mathrm{C}$. Experiments by Saunders et al. [뜨] showed that the warming effect of a riming target is sufficient to reduce its growth rate by vapour deposition below that of the rebounding ice crystals, resulting in the graupel target acquiring negative charge. Thus, during ice crystal-graupel interactions, that the ice crystal grows faster at colder temperatures is understandable, and the riming graupel charges negatively even if it is the warmer of the interacting particles, with an opposite temperature effect to that required by the surface tension gradient mechanism.

\subsection{Thermoelectric effect and Surface tension gradient}

The mechanism based on the relative growth rate of cloud particles is able to explain the charge reversal of growth phenomena, where the vapour-grown crystals grow faster than graupel and charge positively at colder temperatures, while graupel grow faster and charge negatively at warmer temperatures. However, how the relative growth rates of these cloud particles would vary with humidity and temperature, and how do the charge polarity of particles relate to the growth rates are unclear. The thermoelectric effect and surface tension gradient also explain charge transfer in particle collisions, but their applicability is limited to lower and higher humidty atmospheric conditions, respectively [22]. In fact, the amount of water vapour in the troposphere generally decreases with height [47] The thermoelectric effect and surface tension gradient would play a leading role in different altitude of thundercloud, respectively.

In the lower (warmer) region of thunderclouds, liquid water content is high and ice particles tend to be in wet growth. For an ice particle with liquid water film formed by vapour diffusion, the gas-water interface would accumulate more negative charge due to the release of potential heat. The ice-water interface also charges negatively according to the double-layer stable theory. Thus, the thick stable water films covering the ice particles will be stabilized by electrostatic repulsion between negatively charged ice-water and gas-water interfaces [22]. A rapidly growing surface tends to acquire a thicker liquid layer, a higher temperature and more negative charge [표]. Due to ice crystalgraupel interactions, the graupel will lose negative charge according to two mechanisms: (i) The surface tension gradient-driven mechanism where the interfacial flow is drawn from the higher temperature body to the lower temperature body, and (ii) the thermal diffusion mechanism where ions diffuse from regions of higher concentration to regions of lower concentration. Charge transfer in these two processes is consistent in direction when particles are in wet growth. The ice crystal ultimately acquires a net negative charge, as shown in Fig. 7.

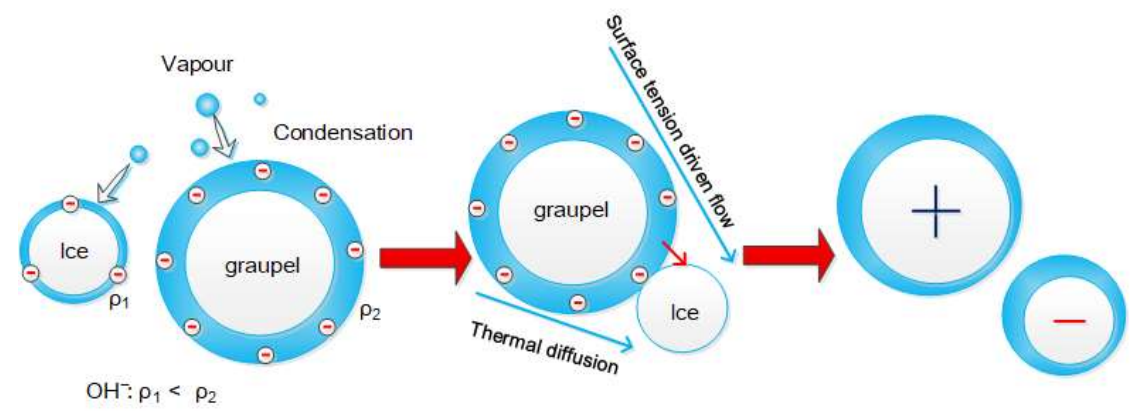

Figure 7. Charge transfer between ice crystal and graupel in wet growth. 
In the higher (colder) region, droplets freeze quickly at lower temperatures. The probability of an ice crystal encountering a fast-growing region around a freezing droplet would be lower. In addition, the liquid-like layer is very thin, meaning that it cannot be explained with the surface tension gradient theory, where water flows must exist. In the dry growth region, the ice crystal with higher growth speed accumulates more negative charges. During the interaction between graupel and ice crystal, the ice crystal will lose some negative charge and the graupel acquires a net negative charge, which are mainly controlled by the diffusion mechanism, as shown in Fig. 8.

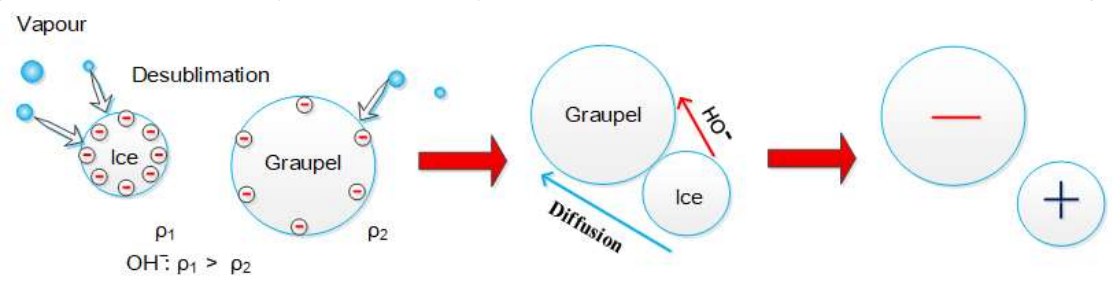

Figure 8. Charge transfer between ice crystal and graupel in dry growth.

Therefore, the combination of thermoelectric effect and surface tension gradient would explain the charge separation and charge reversal in thunderclouds as well, consistent with the relative growth rate mechanism. The distribution of actual atmospheric humidity is non-uniformly varying in vertical altitude, and horizontal humidity gradients could exist due to strong updraft-downdraft in thunderclouds. So more complex charge structure would be formed under the combined electrical mechanism.

\subsection{Other influence factors}

In addition, many researchers have suggested that charge is also related to particle size, particle shape, and the relative velocity of collision objects, besides temperature and effective liquid water content of clouds $[\underline{9}, \underline{20}, \underline{49}, \underline{50}]$. First, particle size affects the growth rate of crystals. Large crystals act to diminish somewhat the significance of the rapid transient growth relative to smaller crystals. Relatively faster growing crystals (large or small) promote negative graupel charging (more for small crystals), which is consistent with the relative growth rate theory. Second, changes in particle shape could bring about a reversal of the charge sign. According to the thermoelectric effect, irregular particles with more tips will gain more heat locally, giving rise to a higher temperature. Thus, during collisions between graupel particles and these ice particles, the graupel particles charge positively while the irregular particles carry away negative charges. Third, relative velocity between collision subjects affects the charge amount. A low velocity would lead to a longer contact time, which thus causes further neutralization between particles [ $\underline{51}, \underline{52}]$. In addition, at a higher relative velocity, the particles will experience a higher temperature change in a short time, breaking the thermal equilibrium inside the particles. The large temperature difference would cause a higher growth rate, and, consequently increases the charge transfer according to the relative growth rate theory.

\subsection{Non-thermal equilibrium ionization}

Charge separation in ice crystal-graupel collision is driven by the non-thermal equilibrium of the hydrometeors, i.e., non-thermal equilibrium ionization. The non-thermal equilibrium ionization is able to uncover the fundamental essence of relative rate theory, unify the thermoelectric effect and surface tension gradient, and explain other complex charge separation. For charge migration inside hydrometeors, the temperature gradient-driven mechanism is to maintain the thermal equilibrium between the hydrometeor and environment. The chemical potential gradient-driven mechanism is to maintain the chemical equilibrium and its effect on charge migration is very limited when at a constant temperature. When temperature increases, ion concentrations will be elevated, and thus the charge migration due to the chemical potential gradient-driven will be correspondingly enhanced. For charge separation between colliding hydrometeors, the relative growth rate theory is based on the fact that the transient rapid growth occurs when the thermal equilibrium of the crystal is broken. 
The thermoelectric effect and the surface tension gradient are driven by the temperature differential. The particle size can affect the growth rate (and thus temperature) of ice particles. The particle shape can influence heat transfer and thus can generate a temperature differential. The relative velocity can cause the particle to experience different atmospheric temperatures in a limited time, and this would affect the growth rate.

In summary, the charge migration inside hydrometeor particles in principal depends on temperature differential between particles and the environment. The subsequent charge separation between colliding hydrometeors depends on the electric state of the surfaces. Therefore, the fundamental cause of the ice crystal-graupel collision charging is that these particles are in a nonthermal equilibrium state. In other words, thunderstorm is a transient and rapid-development phenomenon, where cloud particles are strongly moving upward-downward. The relaxation time needed to reach a new thermal equilibrium through conduction is thus relatively longer, resulting in most cloud particles being in non-thermal equilibrium. Based on these results, we conclude that thunderstorm electrification is actually a dynamic, non-thermal equilibrium process of cloud particles.

\section{Conclusions and perspectives}

The charging of ice crystal-graupel collision could play an important role in thunderstorm electrification, whether in the initial or developing stages of thunderstorms. Ion migration inside hydrometeors is driven by the temperature gradient and the chemical potential gradient, and the combined action of these two driving forces determines the surface electrical states of hydrometeors. When the hydrometeor is in thermal equilibrium, a small amount of net negative charge would be accumulated on the surface due to the chemical potential gradient. As the particle temperature increases, the chemical potential of $\mathrm{H}^{+} / \mathrm{OH}^{-}$will be enhanced, leading to more excess negative ions on the surface. Therefore, the effect of the chemical potential gradient on charge migration is due to the temperature gradient.

By analyzing relative growth rate theory, the thermoelectric effect (charge transfer) and the surface tension gradient (mass transfer along with charge transfer), we propose that the relative growth rates theory could properly explain the charge separation in thunderstorms, while the thermoelectric effect and the surface tension gradient are applicable in specific environmental conditions. The combined action of the thermoelectric effect and the surface tension gradient is consistent with the relative growth rate theory. Then, the influences of particle size, particle shape and relative velocity on charge separation are analyzed as a complement to the relative growth rate mechanism, which is generally controlled by temperature and relative humidity. Thus, temperature and humidity are the key factors that impact the charge separation during the ice crystal-graupel collisions. However, they are dynamically changed with the development of thunderstorms, so the dynamic processes of microphysical conditions should be considered in future research on thunderstorm electrification, especially the non-thermal equilibrium of cloud particles. Furthermore, water droplets in clouds could also experience non-thermal equilibrium and subsequent charge transfer as ice particles, so the mechanisms presented here could also be extended to explain warm cloud lightning.

\section{Acknowledgments}

This work was supported by the National Natural Science Foundation of China, grant number 11872295; the National Key R\&D Program of China, grant number 2016ZX05011001; the Strategic Research Grants of City University of Hong Kong, grant number SRG-7004867; and the Innovation and Technology Fund (ITF) of HKSAR, grant number ITS/410/16FP.

\section{Conflicts of Interest}

There is no potential conflict of interest in the preparation or in the publication of this paper. 


\section{References}

1. Solomon, R.; Baker, M. Electrification of New Mexico Thunderstorms. Mon. Weather Rev. 1994, 122, 34473459 .

2. Vonnegut, B.; Latham, D.J.; Moore, C.B.; Hunyady, S.J. An explanation for anomalous lightning from forest fire clouds. J. Geophys. Res. 1995, 100, 5037-5050.

3. Mason, J. The Generation of Electric Charges and Fields in Thunderstorms. P. Roy. Soc. A 1988, 415, 303315.

4. Kuettner, J.P.; Doyne Sartor, J.; Levin, Z. Thunderstorm Electrification--Inductive or Non-Inductive? J. Atmos. Sci. 1981, 38, 2470-2484.

5. Dye, J.E.; Jones, J.J.; Weinheimer, A.J., Winn, W.P. Observations within two regions of charge during initial thunderstorm electrification. Q. J. Roy. Meteor. Soc. 1988, 114, 1271-1290.

6. Zheng, G.; List, R. Convective heat transfer of rotating spheres and spheroids with non-uniform surface temperatures. Int. J. Heat Mass Tran. 1996, 39, 1815-1826.

7. Bruning, E.C.; Rust, W.D.; Schuur, T.J.; Macgorman, D.R.; Krehbiel, P.R.; Rison, W. Electrical and Polarimetric Radar Observations of a Multicell Storm in TELEX. Mon. Weather Rev. 2007, 135, 2525-2544.

8. Latham, J.; Petersen, W.A.; Deierling, W.; Christian, H.J. Field identification of a unique globally dominant mechanism of thunderstorm electrification. Q. J. Roy. Meteor. Soc. 2007, 133, 1453-1457.

9. Emersic, C.; Saunders, C.P.R. Further laboratory investigations into the Relative Diffusional Growth Rate theory of thunderstorm electrification. Atmos. Res. 2010, 98, 327-340.

10. Luque, M.; Bürgesser, R., and Ávila, E. Thunderstorm graupel charging in the absence of supercooled water droplets. Q. J. Roy. Meteor. Soc. 2016, 142, 2418-2423.

11. Shuang, X.U.; Zheng, D.; Wang, Y.; Pengyu, H.U., et al., Characteristics of the Two Active Stages of Lightning Activity in Hailstorms. J. Meteor. Res. 2016, 30, 265-281.

12. Reynolds, S.; Brook, M. Correlation of the initial electric field and the radar echo in thunderstorms. J. Meteor. 1956, 13, 376-380.

13. Qie, X.S.;, Zhang, Y.J.; Yuan, T.; Zhang, Q.L.; Zhang, T.L., et al., A review of atmospheric electricity research in China. Adv. Atmos. Sci. 2015, 32, 169.

14. Sun, J.; Guo, F.X. Effect of cloud saturation on non-inductive electrification process in thunderstorm. Trans. Atmos. Sci. 2015, 93.

15. Williams, E.R. The tripole structure of thunderstorms. J. Geophys. Res.: Atmos, 1989, 94, 13151-13167.

16. Zhang, Y.J.; Dong, W.S.; Zhao, Y.; Zhang, G.S.; Zhang, H.F., et al. Study of charge structure and radiation characteristic of intracloud discharge in thunderstorms of Qinghai-Tibet Plateau. Sci. China Ser. D 2004, 47, 108-114.

17. Zhao, Z.K.; Qie, X.S.; Zhang, T.L.; Zhang, T.; Zhang, H.F., et al. Electric field soundings and the charge structure within an isolated thunderstorm. Chinese Sci. Bull. 2010, 55, 872-876.

18. Jayaratne, E.R.; Saunders, C.P.R.; Hallett, J. Laboratory studies of the charging of soft-hail during ice crystal interactions. J. Roy. Meteor. Soc. 1983, 109, 609-630.

19. Williams, E.R. Thunderstorm Electrification: Precipitation Versus Convection. Ph.D. Thesis, Massachusetts Institute of Technology, Massachusetts, 1981.

20. Jayaratne, E.R.; Saunders, C.P.R. The interaction of ice crystals with hailstones in wet growth and its possible role in thunderstorm electrification. Q. J. Roy. Meteor. Soc. 2016, 142, 1809-1815.

21. Takahashi, T. Thermoelectric effect in ice. J. Atmos. Sci. 1966, 23, 74-77.

22. Graciaa, A., Creux, P., Lachaise, J., Schechter, R.S. Charge transfer between colliding hydrometeors: Role of surface tension gradients. J. Geophys. Res. 2001, 106, 7967-7972.

23. Schechter, R.S.; Graciaa, A.; Lachaise, J. The Electrical State of a Gas/Water Interface. J. Colloid Interf. Sci. 1998, 204, 398-9.

24. Sato, H.; Yui, M.; Yoshikawa, H. Ionic Diffusion Coefficients of Cs+, Pb2+, Sm3+, Ni2+, SeO2-4 and TcO4 in Free Water Determined from Conductivity Measurements. J. Nucl. Sci. Technol. 1996, 33, 950-955.

25. Latham, J.; Mason B.J. Electric Charge Transfer Associated with Temperature Gradients in Ice. P. Royal Soc. London. A 1961, 260, 523-536.

26. Latham, J. Charge Transfer associated with Temperature Gradients in Ice. Nature 1963, 200, 1087-1087.

27. MacGorman, D.R.; Rust, W.D.; MacGorman, R.; Rust, W. The electrical nature of storms; Oxford University Press: New York, USA, 1998; pp. 60-61, 165.

28. Mason, J.A.; de Blij H. Physical Geography: The Global Environment. Oxford University Press: New York, 
USA, 2015.

29. Israelachvili, J. Intermolecular and surface forces (in Chinese), 3rd ed.; Elsevier: New York, USA, 2012; pp. $25,56,120$.

30. Fletcher, N. Surface structure of water and ice: II. A revised model. Philos. Mag. 1968, 18, 1287-1300.

31. Jayaratne, R. Thunderstorm electrification mechanisms. In The Lightning Flash, 2nd ed.; Cooray, V. Eds.; IET Digital Library, 2014; pp. 25-30.

32. Hobbs, P.V. Ice physics. Clarendon Press: Clarendon, USA, 1974; pp. 873.

33. Keith, W.D.; Saunders, C.P.R. Further laboratory studies of the charging of graupel during ice crystal interactions. Atmos. Res. 1990, 25, 445-464.

34. Dare, R.A.; Davidson N.E. Seasonal distributions of daily rain events over Australia. Bureau of Meteorology, Victoria, 2015; pp. 5-25.

35. Gu, Z.L., Wei, W., Su, J., Yu, C.W. The role of water content in triboelectric charging of wind-blown sand. Sci. Rep. 2013, 3, 1337.

36. Wei, W.; Gu, Z. Electrification of particulate entrained fluid flows-mechanisms, applications, and numerical methodology. Phys. Rep. 2015, 600, 1-53.

37. Houze Jr, R.A. Cloud microphysics. In Cloud dynamics; Renata, D, James R. H Eds.; Academic press: New York, USA, 2014; pp. 73-75.

38. Committee, G.S.; Council, N.R. The Earth's Electrical Environment; National Academies Press: Washington, USA, 1986; pp. 121-126.

39. Amp, J.M.C.; Illingworth, A.J. Surface potentials of ice and thunderstorm charge separation. Nature 1980, 284, 44-46.

40. Lamb, D.; Verlinde, J. Physics and Chemistry of Clouds; Cambridge University Press: London, UK, 2011.

41. Saunders, C.P.R., et al., A laboratory study of the influence of ice crystal growth conditions on subsequent charge transfer in thunderstorm electrification. Q. J. Roy. Meteor. Soc. 2006, 130, 1395-1406.

42. Pruppacher, H.R.; Klett, J.D. Microphysics of Clouds and Precipitation: Reprinted 1980; Springer Science \& Business Media, 2012.

43. Saunders, C.P.R.; Bax-Norman, H.; Emersic, C.; Avila, E.E.; Castellano, N.E. Laboratory studies of the effect of cloud conditions on graupel/crystal charge transfer in thunderstorm electrification. Q. J. Roy. Meteor. Soc. 2006, 132, 2653-2673.

44. Bolot, M.; Legras, B.; Moyer, E.J. Modelling and intepreting the isotopic composition of water vapour in convective updrafts. Atmos. Chem. Phys. 2013, 13, 7903-7935.

45. Arnold, L.; Glassmeier, F.; Dietlicher, R.; Paukert, M.; Lohmann, U. Importance of realistic ice crystal radii for charge generation according to the Relative Diffusional Growth Rate theory, Paper presented at: EGU General Assembly, Vienna, Austria, 23-28 April 2017; EGU: Vienna, Austria, 2017; 19, EGU201711572.

46. Saunders, C.P.R.; Keith, W.D.; Mitzeva, R.P. The effect of liquid water on thunderstorm charging. J. Geophys. Res. Atmos. 1991, 96, 11007-11017.

47. Sheng, P. Atmospheric Physics: Composition and distribution of the earth's atmosphere (in Chinese); Peking University Press: Beijing, China, 2003.

48. Saunders, C.P.R.; Bax-Norman, H.; Avila, E.E.A.; Castellano, N.E. A laboratory study of the effects of rime ice accretion and heating on charge transfer during ice crystal/graupel collisions. Atmos. Res. 1999, 51, 99-117.

49. Avila, E.E.; Saunders, C.P.R.; Bax-Norman, H.; Castellano, N.E. Charge sign reversal in irregular ice particle-graupel collisions. Geophys. Res. Lett. 2005, 32, 357-357.

50. Brooks, I.M.; Saunders, C.P.R. An experimental investigation of the inductive mechanism of thunderstorm electrification. J. Geophys. Res. A. 1994, 99, 10627-10632.

51. Marshall, B.J.P.; Latham, J.; Saunders, C.P.R. A laboratory study of charge transfer accompanying the collision of ice crystals with a simulated hailstone. Q. J. Roy. Meteor. Soc. 1978, 104, 163-178.

52. Keith, W.D.; Saunders, C.P.R. Light emission from colliding ice particles. Nature 1988, 336, 362-364. 